\title{
Symmetries for Siegel Theta Functions, Borcherds Lifts and Automorphic Green Functions
}

\author{
Bernhard Heim and Atsushi Murase
}

2000 Mathematics Subject Classification numbers: primary 11F55, secondary 11G18

\begin{abstract}
Let $q$ be an integral quadratic form of signature $(2, m+2)$. We will show that the Siegel theta functions attached to $q$ satisfies certain symmetries. As an application, we prove the symmetries for automorphic forms on the orthogonal group of $q$ closely related to Heegener divisors (Borcherds lifts and automorphic Green functions).
\end{abstract}

\section{Introduction}

\subsection{The main results}

Let $(L, q)$ be a quadratic space over $\mathbb{Z}$ of signature $\left(b^{+}, b^{-}\right)$with $b^{-} \geq b^{+}>0$, and $G$ the orthogonal group of $q$. Let $\operatorname{Gr}(L)$ be the set of $b^{+}$-dimensional subspaces $v$ of $V=L \otimes_{\mathbb{Z}} \mathbb{R}$ such that $\left.q\right|_{v}>0$. The Grassmannian $\operatorname{Gr}(L)$ of $L$ is a real analytic manifold on which $G(\mathbb{R})$ acts in a natural manner. For $\lambda \in V$ and $v \in \operatorname{Gr}(L)$, we denote by $\lambda_{v}$ and $\lambda_{v}^{\perp}$ the projections of $\lambda$ to $v$ and $v^{\perp}$ respectively, where $v^{\perp}$ is the orthogonal complement of $v$ in $V$ with respect to $q$. Let $L^{*}$ be the dual lattice of $L$ and let $\mathfrak{H}=\{\tau \in \mathbb{C} \mid \operatorname{Im}(\tau)>0\}$ be the upper half plane. For $\alpha \in L^{*} / L$ and $(\tau, v) \in \mathfrak{H} \times \operatorname{Gr}(L)$, we define the Siegel theta function by

$$
\Theta_{\alpha}(\tau, v)=\sum_{\lambda \in \alpha+L} \exp \left(2 \pi i\left(\tau q\left(\lambda_{v}\right)+\bar{\tau} q\left(\lambda_{v}^{\perp}\right)\right)\right) .
$$

The Siegel theta function, first introduced by Siegel ([Si]), is of fundamental importance in number theory. In particular it plays an crucial role in the arithmetic of quadratic forms and the theory of automorphic forms of several variables.

From now on we assume that $b^{+}=2$, in which case $\operatorname{Gr}(L)$ has a structure of hermitian symmetric domain of type IV. The object of the paper is to show that certain symmetries hold for the Siegel theta functions. As an application of this result, we obtain similar symmetries for automorphic forms on $\operatorname{Gr}(L)$ closely related to Heegner divisors (Borcherds lifts and automorphic Green functions).

To state our main results more precisely, let $m$ be a nonnegative integer and $S$ an even integral positive definite symmetric matrix of degree $m$. Let $L=\mathbb{Z}^{m+4}$ and $q$ a quadratic form given by

$$
q(x)=\frac{1}{2}{ }^{t} x Q x \quad(x \in L),
$$


where

$$
Q=\left(\begin{array}{lllll} 
& & & & 1 \\
& & & 1 & \\
& & -S & \\
& 1 & & & \\
1 & & & &
\end{array}\right)
$$

Note that we include the case of $m=0$, in which case $q(x)=x_{1} x_{4}+x_{2} x_{3}$ for $x={ }^{t}\left(x_{1}, x_{2}, x_{3}, x_{4}\right) \in$ $\mathbb{Z}^{4}$.

It is known that $\operatorname{Gr}(L)$ is isomorphic to the tube domain $\mathcal{D}=\left\{\left(z, w, z^{\prime}\right) \in \mathfrak{H} \times \mathbb{C}^{m} \times \mathfrak{H} \mid\right.$ $\left.2 \operatorname{Im}(z) \operatorname{Im}\left(z^{\prime}\right)-{ }^{t} \operatorname{Im}(w) S \operatorname{Im}(w)>0\right\}$. We henceforth consider $\Theta_{\alpha}$ as a function on $\mathfrak{H} \times \mathcal{D}$. The main result of the paper is the following additive symmetry for Siegel theta functions.

Theorem 1. For $\alpha \in L^{*} / L$ and a natural number $n$, we have

$$
\sum_{a, b, d} \Theta_{\alpha}\left(\tau,\left(\frac{a z+b}{d}, \frac{\sqrt{n}}{d} w, z^{\prime}\right)\right)=\sum_{a, b, d} \Theta_{\alpha}\left(\tau,\left(z, \frac{\sqrt{n}}{d} w, \frac{a z^{\prime}+b}{d}\right)\right),
$$

where $(a, b, d)$ runs over the set $\mathcal{H}_{n}=\left\{(a, b, d) \in \mathbb{Z}^{3} \mid a, d>0, a d=n, 0 \leq b<d\right\}$.

The notion of the additive symmetry was first introduced by the first named author in the Siegel modular case $([\mathrm{H}])$. In $[\mathrm{HeMu}$, we defined the additive symmetry for automorphic forms on $O(2, m+2)$ and showed that the Maass space is characterized by the additive symmetry.

To state applications of Theorem 1, we recall the definition of Heegner divisors. For $\lambda \in V$, let $D(\lambda)$ be the divisor on $\mathcal{D}$ corresponding to $\{v \in \operatorname{Gr}(L) \mid Q(\lambda, v)=0\}$. If $q(\lambda)<0, D(\lambda)$ is isomorphic to a hermitian symmetric domain of type IV associated with $\left.q\right|_{\lambda^{\perp}}$, where $\lambda^{\perp}$ denotes the orthogonal complement of $\lambda$ in $V$ with respect to $q$. For $\alpha \in L^{*} / L$ and $n \in q(\alpha)+\mathbb{Z}$ with $n<0$, let

$$
H(\alpha, n)=\sum_{\lambda \in \alpha+L, q(\lambda)=n} D(\lambda)
$$

be a divisor on $\mathcal{D}$. Then $H(\alpha, n)$ is invariant under the action of $\Gamma^{*}(L)=\left\{\gamma \in G(\mathbb{R})^{+} \mid \gamma L=\right.$ $\left.L,\left.\gamma\right|_{L^{*} / L}=\mathrm{Id}\right\}$, and defines an algebraic divisor on $\Gamma^{*}(L) \backslash \mathcal{D}$. Here $G(\mathbb{R})^{+}$denotes the identity component of $G(\mathbb{R})$. The Heegner divisor $H(\alpha, n)$ plays an important role in the arithmetic of Shimura varieties attached to $G$ (for example see [HZ], GZ, [vdG]).

Borcherds ([Bo2] $)$ constructed a meromorphic automorphic form on $\Gamma^{*}(L)$ whose divisor is a linear combination of Heegner divisors with coefficients in $\mathbb{Z}$. Such an automorphic form is called a Borcherds lift. A Borcherds lift $\Psi$ is obtained as, essentially, the exponential of a regularized integral of a weakly holomorphic modular form (for a precise definition, see 5.3) against the Siegel theta function. Thus Theorem 1 implies the following multiplicative symmetry for Borcherds lifts:

Corollary 2. Let $\Psi$ be a Borcherds lift. Then we have

$$
\prod_{a, b, d} \Psi\left(\frac{a z+b}{d}, \frac{\sqrt{n}}{d} w, z^{\prime}\right)=\epsilon_{n}(\Psi) \prod_{a, b, d} \Psi\left(z, \frac{\sqrt{n}}{d} w, \frac{a z^{\prime}+b}{d}\right)
$$


for any natural number $n$. Here $(a, b, d)$ runs over $\mathcal{H}_{n}$ and $\epsilon_{n}(\Psi)$ is a complex number of absolute value 1 depending on $n$ and $\Psi$.

Finally let $\Phi_{\alpha, n}$ denote the automorphic Green function associated with the Heegner divisor $H(\alpha, n)$, which has been introduced and studied by Bruinier ([Br1], $\mathrm{Br} 2])$ and Oda and Tsuzuki ([OT]) independently (for a precise definition of $\Phi_{\alpha, n}$, see 6.1). Bruinier ([Br2]) showed that $\Phi_{\alpha, n}$ is obtained as a regularized integral of a certain Poincaré series against the Siegel theta function. Thus, again, Theorem 1 implies

Corollary 3. The automorphic Green function $\Phi_{\alpha, n}$ satisfies the additive symmetry.

Remark. The above results (Corollaries 2 and 3) suggest that certain symmetries should hold for Heegner divisors. We hope to study these symmetries in future.

Remark. In this paper, we assume that the Witt index of $q$ is equal to 2 and hence the $\mathbb{Q}$-rank of $G$ is equal to 2 . In the forthcoming paper, we will treat the Hilbert modular case $(G=O(2,2)$ of $\mathbb{Q}$-rank 1), which contains some special features.

\subsection{The organization of the paper}

The paper is organized as follows. In Section 2, after recalling the definition of automorphic forms on $O(2, m+2)$, we introduce the notions of the additive symmetry and the multiplicative symmetry. In Section 3, we recall the definition of Siegel theta functions and state the main result of the paper (Theorem 3.1), the additive symmetry for Siegel theta functions. The definition of Heegner divisors is recalled in Section 4. In Section 5 and 6, we prove the multiplicative (respectively additive) symmetry for Borcherds lifts (respectively automorphic Green functions) assuming Theorem 3.1. Section 7 is devoted to the proof of Theorem 3.1, which relies on a formula due to Borcherds (Proposition 7.1) and Poisson summation formula. In the final section, we study several examples of Siegel modular forms of degree 2. In particular, as an application of the multiplicative symmetry for Borcherds lifts, we show that a holomorphic Siegel cusp form of weight 12 on $\mathrm{Sp}_{2}(\mathbb{Z})$, which is unique up to constant multiples, is not a Borcherds lift.

\subsection{Notation}

As usual, we denote by $\mathbb{N}, \mathbb{Z}, \mathbb{Q}, \mathbb{R}$ and $\mathbb{C}$ the set of natural numbers, the ring of rational integers, the field of rational numbers, the field of real numbers and the field of complex numbers respectively. For a real symmetric matrix $T$ of degree $n$, let $T(x, y)={ }^{t} x T y$ and $T[x]=T(x, x)$ for $x, y \in \mathbb{C}^{n}$. We put $\mathbf{e}(z)=\exp (2 \pi i z)$ for $z \in \mathbb{C}$. Denote by $\delta_{i j}$ the Kronecker's delta. For a condition $C$, we put

$$
\delta(C)= \begin{cases}1 & \text { if } C \text { holds } \\ 0 & \text { otherwise }\end{cases}
$$




\section{Automorphic forms on $O(2, m+2)$ and the symmetry}

Throughout the paper, we fix a positive definite even integral symmetric matrix $S$ of degree $m \geq 0$. For $\nu \in \mathbb{Z}_{\geq 0}$, put

$$
Q_{\nu}=\left(\begin{array}{ccc}
0 & 0 & J_{\nu} \\
0 & -S & 0 \\
J_{\nu} & 0 & 0
\end{array}\right)
$$

where $J_{\nu}=\left(\delta_{i, \nu-j+1}\right)_{1 \leq i, j \leq \nu} \in \mathrm{GL}_{\nu}$. Then $Q_{\nu}$ is an even integral symmetric matrix of signature $(\nu, m+\nu)$. Let $L_{\nu}=\mathbb{Z}^{m+2 \nu}, L_{\nu}^{*}=Q_{\nu}^{-1} L_{\nu}, V_{\nu}=L_{\nu} \otimes_{\mathbb{Z}} \mathbb{R}=\mathbb{R}^{m+2 \nu}$ and $V_{\nu, \mathbb{C}}=V_{\nu} \otimes_{\mathbb{R}} \mathbb{C}=\mathbb{C}^{m+2 \nu}$. We often write $Q, L, L^{*}$ and $V$ for $Q_{2}, L_{2}, L_{2}^{*}$ and $V_{2}$ respectively. Thus

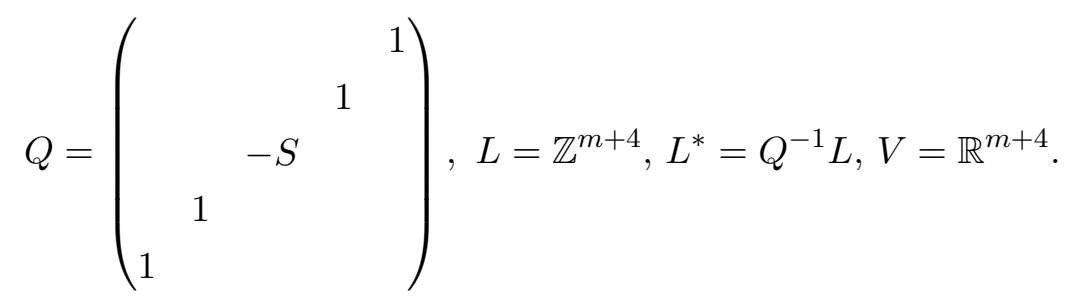

We often write $\left(x_{1}, x_{2}, x_{3}\right)$ for

$$
\left(\begin{array}{l}
x_{1} \\
x_{2} \\
x_{3}
\end{array}\right) \in V_{1, \mathbb{C}} \quad\left(x_{1}, x_{3} \in \mathbb{C}, x_{2} \in V_{0, \mathbb{C}}=\mathbb{C}^{m}\right)
$$

if there is no fear of confusion. Let $q$ be a quadratic form on $V$ defined by $q(X)=2^{-1} Q[X]$ for $X \in V$.

Denote by $G=O(Q)$ the orthogonal group of $Q$. Let $G(\mathbb{R})^{+}$be the identity component of $G(\mathbb{R})$ and

$$
\mathcal{D}=\left\{Z=\left(z, w, z^{\prime}\right) \in V_{1, \mathbb{C}} \mid z, z^{\prime} \in \mathfrak{H}, w \in \mathbb{C}^{m}, Q_{1}[\operatorname{Im}(Z)]=2 \operatorname{Im}(z) \operatorname{Im}\left(z^{\prime}\right)-S[\operatorname{Im}(w)]>0\right\}
$$

As is well-known, $\mathcal{D}$ is a hermitian symmetric domain of type IV. Define an action of $G(\mathbb{R})^{+}$on $\mathcal{D}$ and an automorphic factor $J: G(\mathbb{R})^{+} \times \mathcal{D} \rightarrow \mathbb{C}^{\times}$as follows: For $Z \in \mathcal{D}$, put

$$
\widetilde{Z}=\left(\begin{array}{c}
-Q_{1}[Z] / 2 \\
Z \\
1
\end{array}\right) \in V_{\mathbb{C}}
$$

Note that $Q[\widetilde{Z}]=0$ and $Q(\widetilde{Z}, \bar{Z})=2 Q_{1}[\operatorname{Im}(Z)]>0$. For $g \in G(\mathbb{R})^{+}$and $Z \in \mathcal{D}$, we define $g\langle Z\rangle \in \mathcal{D}$ and $J(g, Z) \in \mathbb{C}^{\times}$by

$$
g \widetilde{Z}=\widetilde{g\langle Z\rangle} J(g, Z) .
$$

Let $k$ be an integer and $F$ a function on $\mathcal{D}$. For $g \in G(\mathbb{R})^{+}$, we define the Petersson slash operator by $\left(\left.F\right|_{k} g\right)(Z)=J(g, Z)^{-k} F(g\langle Z\rangle)$. 
Let $\Gamma(L)=\left\{\gamma \in G(\mathbb{R})^{+} \mid \gamma L=L\right\}$. For an index finite subgroup $\Gamma$ of $\Gamma(L)$, a unitary character $\chi$ of $\Gamma$ and $k \in \mathbb{Z}$, denote by $\mathcal{C}_{k}(\Gamma, \chi)$ the space of smooth functions $F$ on $\mathcal{D}$ satisfying $\left.F\right|_{k} \gamma=\chi(\gamma) F$ for any $\gamma \in \Gamma$.

Define two embeddings of $\mathrm{SL}_{2}$ into $G$ by

$$
\iota^{\uparrow}(h)=\left(\begin{array}{ccccc}
a & & & -b & \\
& a & & & b \\
& & 1_{m} & & \\
-c & & & d & \\
& c & & & d
\end{array}\right), \quad \iota^{\downarrow}(h)=\left(\begin{array}{ccccc}
a & -b & & & \\
-c & d & & & \\
& & 1_{m} & & \\
& & a & b \\
& & & c & d
\end{array}\right)
$$

for $h=\left(\begin{array}{ll}a & b \\ c & d\end{array}\right) \in \mathrm{SL}_{2}$. These embeddings commute with each other, and $\iota^{\uparrow}\left(\mathrm{SL}_{2}(\mathbb{R})\right)$ and $\iota^{\downarrow}\left(\mathrm{SL}_{2}(\mathbb{R})\right)$ are contained in $G(\mathbb{R})^{+}$.

For $F \in \mathcal{C}_{k}(\Gamma, \chi)$ and $n \in \mathbb{N}$, we define

$$
\begin{aligned}
\left.F\right|_{k} T_{n}^{\uparrow}(Z) & =\left.n^{k / 2-1} \sum_{a, b, d} F\right|_{k} \iota^{\uparrow}\left(\sqrt{n}^{-1}\left(\begin{array}{cc}
a & b \\
0 & d
\end{array}\right)\right)(Z) \\
& =\frac{1}{n} \sum_{a, b, d}\left(\frac{n}{d}\right)^{k} F\left(\frac{a z+b}{d}, \frac{\sqrt{n}}{d} w, z^{\prime}\right), \\
\left.F\right|_{k} T_{n}^{\downarrow}(Z) & =\left.n^{k / 2-1} \sum_{a, b, d} F\right|_{k} \iota^{\downarrow}\left(\sqrt{n}^{-1}\left(\begin{array}{cc}
a & b \\
0 & d
\end{array}\right)\right)(Z) \\
& =\frac{1}{n} \sum_{a, b, d}\left(\frac{n}{d}\right)^{k} F\left(\tau,\left(z, \frac{\sqrt{n}}{d} w, \frac{a z^{\prime}+b}{d}\right)\right),
\end{aligned}
$$

where $Z=\left(z, w, z^{\prime}\right) \in \mathcal{D}$ and $(a, b, d)$ runs over

$$
\mathcal{H}_{n}=\left\{(a, b, d) \in \mathbb{Z}^{3} \mid a, d>0, a d=n, 0 \leq b<d\right\}
$$

Note that $\left.F\right|_{k} T_{n}^{\uparrow}$ and $\left.F\right|_{k} T_{n}^{\downarrow}$ are not in $\mathcal{C}_{k}(\Gamma, \chi)$ in general. For a prime $p$, we have

$$
\left.F\right|_{k} T_{p}^{\uparrow}\left(z, w, z^{\prime}\right)=p^{k-1} F\left(p z, \sqrt{p} w, z^{\prime}\right)+p^{-1} \sum_{a=0}^{p-1} F\left(p^{-1}(z+a), \sqrt{p}^{-1} w, z^{\prime}\right)
$$

and

$$
\left.F\right|_{k} T_{p}^{\downarrow}\left(z, w, z^{\prime}\right)=p^{k-1} F\left(z, \sqrt{p} w, p z^{\prime}\right)+p^{-1} \sum_{a=0}^{p-1} F\left(z, \sqrt{p}^{-1} w, p^{-1}\left(z^{\prime}+a\right)\right) .
$$

We say that $F \in \mathcal{C}_{k}(\Gamma, \chi)$ satisfies the additive symmetry if the equality

$$
\left.F\right|_{k} T_{n}^{\uparrow}=\left.F\right|_{k} T_{n}^{\downarrow}
$$

holds for any $n \geq 1$. It is easily seen that $F$ satisfies the additive symmetry if and only if $F$ satisfies (2.1) for $n=p, p$ any prime number. 
We also define

$$
\begin{aligned}
F \mid \mathcal{T}_{n}^{\uparrow}(Z) & =\prod_{a, b, d} F\left(\iota^{\uparrow}\left(\sqrt{n}^{-1}\left(\begin{array}{cc}
a & b \\
0 & d
\end{array}\right)\right)\langle Z\rangle\right) \\
& =\prod_{a, b, d} F\left(\frac{a z+b}{d}, \frac{\sqrt{n}}{d} w, z^{\prime}\right), \\
F \mid \mathcal{T}_{n}^{\downarrow}(Z) & =\prod_{a, b, d} F\left(\iota^{\downarrow}\left(\sqrt{n}^{-1}\left(\begin{array}{cc}
a & b \\
0 & d
\end{array}\right)\right)\langle Z\rangle\right) \\
& =\prod_{a, b, d} F\left(z, \frac{\sqrt{n}}{d} w, \frac{a z^{\prime}+b}{d}\right),
\end{aligned}
$$

where $Z=\left(z, w, z^{\prime}\right) \in \mathcal{D}$ and $(a, b, d)$ runs over $\mathcal{H}_{n}$. We say that $F$ satisfies the multiplicative symmetry if

$$
F\left|\mathcal{T}_{n}^{\uparrow}=\epsilon_{n}(F) F\right| \mathcal{T}_{n}^{\downarrow}
$$

holds for any $n \geq 1$ with a complex number $\epsilon_{n}(F)$ of absolute value 1 depending on $n$ and $F$. Similarly as above, $F$ satisfies the multiplicative symmetry if and only if $F$ satisfies (2.2) for $n=p, p$ any prime number.

\section{Siegel theta functions}

\subsection{The Grassmannian}

Recall that the Grassmannian $\operatorname{Gr}(L)$ of $L$ is the set consisting of 2-dimensional subspaces of $V$ on which $q$ is positive definite. Then $\operatorname{Gr}(L)$ is a hermitian symmetric domain of type IV on which $G(\mathbb{R})^{+}$acts transitively in a natural manner, and isomorphic to $\mathcal{D}$. The isomorphism from $\mathcal{D}$ to $\operatorname{Gr}(L)$ is given by $Z \in \mathcal{D} \mapsto v_{Z}$, the subspace of $V$ generated by $\operatorname{Re}(\widetilde{Z})$ and $\operatorname{Im}(\widetilde{Z})$.

\subsection{Siegel theta functions}

For $\lambda \in V$ and $v \in \operatorname{Gr}(L)$, let $\lambda_{v}$ and $\lambda_{v}^{\perp}$ be the projections of $\lambda$ to $v$ and $v^{\perp}$ respectively, where $v^{\perp}$ denotes the orthogonal complement of $v$ in $V$ with respect to $q$. Then $q(\lambda)=q\left(\lambda_{v}\right)+q\left(\lambda_{v}^{\perp}\right)$. For $\alpha \in L^{*} / L$, the Siegel theta function $\Theta_{\alpha}$ is defined by

$$
\begin{aligned}
\Theta_{\alpha}(\tau, v) & =\sum_{\lambda \in \alpha+L} \mathbf{e}\left(\tau q\left(\lambda_{v}\right)+\bar{\tau} q\left(\lambda_{v}^{\perp}\right)\right) \\
& =\sum_{\lambda \in \alpha+L} \mathbf{e}\left(i \operatorname{Im}(\tau) Q\left[\lambda_{v}\right]+\frac{\bar{\tau}}{2} Q[\lambda]\right)
\end{aligned}
$$

for $\tau \in \mathfrak{H}$ and $v \in \operatorname{Gr}(L)$. As a function of $v, \Theta_{\alpha}(\tau, v)$ is invariant under

$$
\Gamma^{*}(L)=\left\{\gamma \in \Gamma(L) \mid \gamma \lambda \equiv \lambda(\bmod L) \text { for any } \lambda \in L^{*}\right\}
$$


the discriminant group of $L$. For the automorphy of $\Theta_{\alpha}(\tau, v)$ with respect to $\tau$, see 5.2. By abuse of notation, we often write $\Theta_{\alpha}(\tau, Z)$ for $\Theta_{\alpha}\left(\tau, v_{Z}\right)$. Note that $\Theta_{\alpha}(\tau, Z) \in \mathcal{C}_{0}\left(\Gamma^{*}(L), \mathbf{1}\right)$ as a function of $Z \in \mathcal{D}$, where 1 stands for the trivial character of $\Gamma^{*}(L)$. We easily see that

$$
\Theta_{\alpha}(\tau, Z)=\sum_{\lambda \in \alpha+L} \mathbf{e}\left(i \operatorname{Im}(\tau) \frac{|Q(\lambda, \widetilde{Z})|^{2}}{Q_{1}[\operatorname{Im}(Z)]}+\frac{\bar{\tau}}{2} Q[\lambda]\right)
$$

The main result of the paper is stated as follows.

Theorem 3.1. Let $\alpha \in L^{*} / L$ and $\tau \in \mathfrak{H}$. Then $Z \mapsto \Theta_{\alpha}(\tau, Z)$ satisfies the additive symmetry. Namely, for any $n \in \mathbb{N}$, we have

$$
\sum_{a, b, d} \Theta_{\alpha}\left(\tau,\left(\frac{a z+b}{d}, \frac{\sqrt{n}}{d} w, z^{\prime}\right)\right)=\sum_{a, b, d} \Theta_{\alpha}\left(\tau,\left(z, \frac{\sqrt{n}}{d} w, \frac{a z^{\prime}+b}{d}\right)\right),
$$

where $(a, b, d)$ runs over $\mathcal{H}_{n}$.

We postpone the proof of the theorem until Section 7 .

\section{Heegner divisors}

The quotient $\mathcal{X}_{L}=\Gamma^{*}(L) \backslash \mathcal{D}$ is a quasi-projective algebraic variety over $\mathbb{C}$ of dimension $m+2$. For $\lambda \in V$ with $q(\lambda)<0$, let

$$
D(\lambda)=\{Z \in \mathcal{D} \mid Q(\lambda, \widetilde{Z})=0\}
$$

be a complex analytic divisor on $\mathcal{D}$. For $\alpha \in L^{*} / L$ and $n \in q(\alpha)+\mathbb{Z}$ with $n<0$, define

$$
H(\alpha, n)=\sum_{\lambda \in L+\alpha, q(\alpha)=n} D(\lambda)
$$

Then $H(\alpha, n)$ is a $\Gamma^{*}(L)$-invariant divisor on $\mathcal{D}$, called the Heegner divisor of discriminant $(\alpha, n)$. It is known that $H(\alpha, n)$ is the inverse image under the canonical projection of an algebraic divisor on $\mathcal{X}_{L}$, also denoted by $H(\alpha, n)$ (for example see [Br2], 2.2).

\section{Borcherds lifts}

In this section, we recall the definition of Borcherds lifts after [Bo2] and [Br2], and prove the multiplicative symmetry for Borcherds lifts assuming Theorem 3.1 .

\subsection{Metaplectic representations}

Let $\mathrm{Mp}_{2}(\mathbb{R})$ be the metaplectic group. By definition, $\mathrm{Mp}_{2}(\mathbb{R})$ consists of $(M, \phi)$, where $M \in$ $\mathrm{SL}_{2}(\mathbb{R})$ and $\phi$ is a holomorphic function on $\mathfrak{H}$ satisfying $\phi(\tau)^{2}=j(M, \tau)$, and the product is given by $\left(M_{1}, \phi_{1}(\tau)\right)\left(M_{2}, \phi_{2}(\tau)\right)=\left(M_{1} M_{2}, \phi_{1}\left(M_{2}\langle\tau\rangle\right) \phi_{2}(\tau)\right)$. Here

$$
M\langle\tau\rangle=\frac{a \tau+b}{c \tau+d}, j(M, \tau)=c \tau+d \quad\left(M=\left(\begin{array}{ll}
a & b \\
c & d
\end{array}\right) \in \mathrm{SL}_{2}(\mathbb{R}), \tau \in \mathfrak{H}\right)
$$


as usual. Let $\mathrm{Mp}_{2}(\mathbb{Z})$ be the inverse image of $\mathrm{SL}_{2}(\mathbb{Z})$ under the natural projection $\mathrm{Mp}_{2}(\mathbb{R}) \rightarrow$ $\mathrm{SL}_{2}(\mathbb{R})$. It is known that $\mathrm{Mp}_{2}(\mathbb{Z})$ is generated by

$$
T=\left(\left(\begin{array}{ll}
1 & 1 \\
0 & 1
\end{array}\right), 1\right), S=\left(\left(\begin{array}{cc}
0 & -1 \\
1 & 0
\end{array}\right), \sqrt{\tau}\right) .
$$

Let $\left\{\mathfrak{e}_{\alpha}\right\}_{\alpha \in L^{*} / L}$ be the standard basis of the group ring $\mathbb{C}\left[L^{*} / L\right]$ with $\mathfrak{e}_{\alpha} \mathfrak{e}_{\alpha^{\prime}}=\mathfrak{e}_{\alpha+\alpha^{\prime}}$. Let $\langle$, denote the inner product on $\mathbb{C}\left[L^{*} / L\right]$ defined by $\left\langle\sum_{\alpha} x_{\alpha} \mathfrak{e}_{\alpha}, \sum_{\alpha} y_{\alpha} \mathfrak{e}_{\alpha}\right\rangle=\sum_{\alpha} x_{\alpha} \overline{y_{\alpha}}$, where $\alpha$ runs over $L^{*} / L$. There exists a unitary representation $\rho_{L}$ of $\operatorname{Mp}_{2}(\mathbb{Z})$ on $\mathbb{C}\left[L^{*} / L\right]$ defined by

$$
\begin{aligned}
\rho_{L}(T) \mathfrak{e}_{\alpha} & =\mathbf{e}(q(\alpha)) \mathfrak{e}_{\alpha}, \\
\rho_{L}(S) \mathfrak{e}_{\alpha} & =\frac{\exp \left(\frac{\pi i}{4} m\right)}{\sqrt{\left|L^{*} / L\right|}} \sum_{\beta \in L^{*} / L} \mathbf{e}(-Q(\alpha, \beta)) \mathfrak{e}_{\beta}
\end{aligned}
$$

(see [Bo2], $\S 2$ and $[\mathrm{Br} 2], \mathbf{1 . 1})$.

For a function $f$ on $\mathfrak{H}$ with values in $\mathbb{C}\left[L^{*} / L\right], k \in 2^{-1} \mathbb{Z}$ and $(M, \phi) \in \mathrm{Mp}_{2}(\mathbb{Z})$, we put

$$
\left.f\right|_{k}(M, \phi)(\tau)=\phi(\tau)^{-2 k} \rho_{L}(M, \phi)^{-1} f(M\langle\tau\rangle) .
$$

\subsection{Automorphy of Siegel theta functions}

Set

$$
\Theta_{L}(\tau, Z)=\sum_{\alpha \in L^{*} / L} \mathfrak{e}_{\alpha} \Theta_{\alpha}(\tau, Z)
$$

For $(M, \phi) \in \mathrm{Mp}_{2}(\mathbb{Z})$, we have

$$
\Theta_{L}(M\langle\tau\rangle, Z)=\phi(\tau)^{2} \overline{\phi(\tau)}^{m+2} \rho_{L}(M, \phi) \Theta_{L}(\tau, Z)
$$

(see [Bo2], Theorem 4.1).

\subsection{Weakly holomorphic modular forms}

For $k \in 2^{-1} \mathbb{Z}$, let $\mathcal{M}_{k}\left(\rho_{L}\right)$ be the space of holomorphic functions $f$ on $\mathfrak{H}$ with values in $\mathbb{C}\left[L^{*} / L\right]$ satisfying the following two conditions:

(i) For $(M, \phi) \in \mathrm{Mp}_{2}(\mathbb{Z})$, we have $\left.f\right|_{k}(M, \phi)=f$.

(ii) Let

$$
f(\tau)=\sum_{\alpha \in L^{*} / L, n \in q(\alpha)+\mathbb{Z}} c(\alpha, n) \mathfrak{e}_{\alpha}(n \tau)
$$

be the Fourier expansion of $f$, where we put $\mathfrak{e}_{\alpha}(z)=\mathbf{e}(z) \mathfrak{e}_{\alpha}$ for $z \in \mathbb{C}$. For every $\alpha \in L^{*} / L$, we have $c(\alpha, n)=0$ if $n \ll 0$.

We call $\mathcal{M}_{k}\left(\rho_{L}\right)$ the space of weakly holomorphic modular forms of weight $k$ with respect to $\rho_{L}$. 


\section{4}

Let $f \in \mathcal{M}_{-m / 2}\left(\rho_{L}\right)$. For $N>1$ and $s \in \mathbb{C}$, we put

$$
\Phi_{N}(Z, f, s)=\int_{F_{N}}\left\langle f(\tau), \Theta_{L}(\tau, Z)\right\rangle \operatorname{Im}(\tau)^{-1-s} d \operatorname{Re}(\tau) d \operatorname{Im}(\tau) \quad(Z \in \mathcal{D}),
$$

where $F_{N}=\{\tau \in \mathfrak{H}|| \operatorname{Re}(\tau)|\leq 1 / 2,1 \leq| \tau \mid \leq N\}$. Borcherds ([Bo2] $)$ showed that the limit

$$
\Phi(Z, f, s)=\lim _{N \rightarrow \infty} \Phi_{N}(Z, f, s)
$$

exists if $\operatorname{Re}(s)$ is sufficiently large, and $\Phi(Z, f, s)$ is continued to a meromorphic function of $s$ in $\mathbb{C}$. Denote by $\Phi(Z, f)$ the constant term of the Laurent expansion of $\Phi(Z, f, s)$ at $s=0$. The following fundamental result is due to Borcherds ([Bo2], Theorem 13.3; see also [Br2], 3.4).

Theorem 5.1. Let $f \in \mathcal{M}_{-m / 2}\left(\rho_{L}\right)$ and suppose that

$$
c(\alpha, n) \in \mathbb{Z} \quad \text { for } \alpha \in L^{*} / L \text { and } n \leq 0 .
$$

Then there exists a meromorphic automorphic form $\Psi_{f}(Z)$ on $\Gamma^{*}(L)$ of weight $c(0,0) / 2$ and some multiplier system of $\Gamma^{*}(L)$ of finite order satisfying the following properties.

(i) The divisor of $\Psi_{f}(Z)$ is given by

$$
\frac{1}{2} \sum_{\alpha \in L^{*} / L} \sum_{n \in q(\alpha)+\mathbb{Z}, n<0} c(\alpha, n) H(\alpha, n) .
$$

Here the multiplicities of $H(\alpha, n)$ are 2 (respectively 1 ) if $2 \alpha=0$ (respectively if $2 \alpha \neq 0$ ) in $L^{*} / L$.

(ii) We have

$$
\log \left|\Psi_{f}(Z)\right|=-\frac{1}{4} \Phi(Z, f)-\frac{c(0,0)}{2}\left(\log Q_{1}[\operatorname{Im}(Z)]+\Gamma^{\prime}(1) / 2+\log \sqrt{2 \pi}\right) .
$$

(iii) For each Weyl chamber $W$ with respect to $f$, the product expansion

$$
\Psi_{f}(Z)=C \mathbf{e}\left(Q_{1}\left(Z, \rho_{f}(W)\right)\right) \times \prod_{\lambda \in L_{1}^{*},(\lambda, W)>0}\left(1-\mathbf{e}\left(Q_{1}(\lambda, Z)\right)\right)^{c\left(\widehat{\lambda}, Q_{1}[\lambda] / 2\right)}
$$

holds if $Q_{1}[\operatorname{Im}(Z)] \gg 0$ and $\operatorname{Im}(Z) / \sqrt{Q_{1}[\operatorname{Im}(Z)]} \in W$. Here $C$ is a constant of absolute value $1, \rho_{f}(W) \in V_{1}$ is the Weyl vector attached to $(f, W)$ and $\hat{\lambda}$ is the element of $L^{*} / L$ whose natural projection to $L_{1}^{*} / L_{1}$ is $\lambda+L_{1}$. (For the definitions of Weyl chambers and Weyl vectors, see [Bo2].)

We also have the following converse theorem due to Bruinier ( $\mathrm{Br} 2$, Theorem 5.12).

Theorem 5.2. Let $\Psi$ be a meromorphic automorphic form on $\Gamma^{*}(L)$ whose divisor is a $\mathbb{Z}$ linear combination of Heegner divisors. Then there exists a weakly holomorohic modular form $f \in \mathcal{M}_{-m / 2}\left(\rho_{L}\right)$ satisfying (5.5) such that $\Psi$ is a nonzero constant multiple of $\Psi_{f}$.

We call $\Psi$ satisfying the condition of Theorem 5.2 a Borcherds lift on $\Gamma^{*}(L)$. 


\subsection{Multiplicative symmetry for Borcherds lifts}

Theorem 5.3. Let $\Psi$ be a Borcherds lift on $\Gamma^{*}(L)$. Then $\Psi$ satisfies the multiplicative symmetry.

Proof. We may suppose that $\Psi=\Psi_{f}$ with some $f \in \mathcal{M}_{-m / 2}\left(\rho_{L}\right)$. By Theorem 3.1 and the definition of $\Phi(Z, f), Z \mapsto \Phi(Z, f)$ satisfies the additive symmetry. The multiplicative symmetry for $\Psi_{f}$ follows directly from this fact in view of Theorem 5.1 (ii).

\section{Automorphic Green functions}

\subsection{Automorphic Green functions}

The automorphic Green functions associated with Heegner divisors have been introduced by Bruinier ([Br1], [Br2] $)$ and Oda and Tsuzuki $([\mathrm{OT}])$ independently. Note that the unitary group case is also studied by Oda and Tsuzuki. The automorphic Green functions are also studied by Bruinier and Kühn $([\mathrm{BK}])$ from an arithmetic point of view. In this section, we recall the definition of the automorphic Green functions mainly after [BK], and show their additive symmetry assuming Theorem 3.1 .

Put $\kappa=(m+4) / 2$. Let $\alpha \in L^{*} / L$ and $n \in q(\alpha)+\mathbb{Z}$ with $n<0$. For $Z \in \mathcal{D} \backslash H(\alpha, n)$ and $s \in \mathbb{C}$ with $\operatorname{Re}(s)>\kappa / 2$, we set

$$
\begin{aligned}
\Phi_{\alpha, n}(Z, s)=2 & \frac{\Gamma(s+\kappa / 2-1)}{\Gamma(2 s)} \\
& \times \sum_{\lambda \in \alpha+L, q(\lambda)=n}\left(\frac{n}{n-q\left(\lambda_{Z}\right)}\right)^{s+\kappa / 2-1} F\left(s+\frac{\kappa}{2}-1, s-\frac{\kappa}{2}+1,2 s ; \frac{n}{n-q\left(\lambda_{Z}\right)}\right),
\end{aligned}
$$

where $\lambda_{Z}=\lambda_{v_{Z}}$ and

$$
\begin{aligned}
F(a, b, c ; z) & =\sum_{k=0}^{\infty} \frac{(a)_{k}(b)_{k}}{(c)_{k}} \frac{z^{k}}{k !} \\
(a)_{k} & =\frac{\Gamma(a+k)}{\Gamma(a)} .
\end{aligned}
$$

Note that $q\left(\lambda_{Z}\right)=\left(2 Q_{1}[\operatorname{Im}(Z)]\right)^{-1}|Q(\lambda, \widetilde{Z})|^{2}$. The series (6.1) converges locally uniformly for $Z \in \mathcal{D} \backslash H(\alpha, n)$ and $\operatorname{Re}(s)>\kappa / 2$. It is easy to see that $\Phi_{\alpha, n}(Z, s)$ is $\Gamma^{*}(L)$-invariant in $Z$.

Theorem 6.1 ([Br1], [Br2], $\mathrm{OT}])$. (i) The function $\Phi_{\alpha, n}(Z, s)$ has a meromorphic continuation in $s$ to a neighborhood of $\kappa / 2$ with a simple pole at $s=\kappa / 2$.

(ii) As a function of $Z, \Phi_{\alpha, n}(Z, s)$ is real analytic on $\mathcal{D} \backslash H(\alpha, n)$ and has a logarithmic singularity along $H(\alpha, n)$. Namely, if $U$ is a compact neighborhood of any $Z_{0} \in \mathcal{D}$, there exists a finite set $S(U)$ of $\lambda \in \alpha+L$ with $q(\lambda)=n$ such that

$$
\Phi_{\alpha, n}(Z, s)=-4 \sum_{\lambda \in S(U)} \log q\left(\lambda_{Z}\right)+O(1)
$$


on $U$.

(iii) Let $\Delta$ denote the $G(\mathbb{R})$-invariant Laplace operator on $\mathcal{D}$ induced by the Casimir element of the Lie algebra of $G(\mathbb{R})$ normalized as in [Br2], page 72. Then we have

$$
\Delta \Phi_{\alpha, n}(Z, s)=\Lambda(s) \Phi_{\alpha, n}(Z, s)
$$

where

$$
\Lambda(s)=\frac{1}{2}\left(s-\frac{\kappa}{2}\right)\left(s+\frac{\kappa}{2}-1\right) .
$$

(iv) Suppose that $\operatorname{Re}(s)>\kappa / 2$. Then $\Phi_{\alpha, n}(Z, s) \in L^{1}\left(\mathcal{X}_{L}\right)$. If $f$ is a smooth bounded function on $\mathcal{X}_{L}$ with $\Delta f=\Lambda_{f} f$, then

$$
\int_{\mathcal{X}_{L}} \Phi_{\alpha, n}(Z, s) f(Z) \Omega^{m+2}=-\frac{m+2}{2 \Gamma(s-\kappa / 2+1)} \frac{1}{\Lambda_{f}-\Lambda(s)} \int_{H(\alpha, n)} f(Z) \Omega^{m+1} .
$$

Here $\Omega=-d d^{c} \log Q(\widetilde{Z}, \overline{\widetilde{Z}})$.

We call $\Phi_{\alpha, n}(Z, s)$ the automorphic Green function associated with the Heegner divisor $H(\alpha, n)$.

\subsection{The additive symmetry for automorphic Green functions}

Theorem 6.2. For $\alpha \in L^{*} / L$ and $n \in q(\alpha)+\mathbb{Z}$ with $n<0$, the automorphic Green function $\Phi_{\alpha, n}(Z, s)$ satisfies the additive symmetry in $Z$.

Proof. To prove the theorem, we recall the result of Bruinier $([\mathrm{Br} 2])$. Put $k=-m / 2$ and

$$
\mathbf{M}_{s}(y)=y^{s-k / 2} e^{-y / 2} \sum_{l=0}^{\infty} \frac{(s+k / 2)_{l}}{(2 s)_{l} l !} y^{l} \quad(y>0, s \in \mathbb{C}) .
$$

Define the non-holomorphic Poincaré series

$$
F_{\alpha, n}(\tau, s)=\left.\frac{1}{2 \Gamma(2 s)} \sum_{(M, \phi) \in \widetilde{\Gamma}_{\infty} \backslash M_{p_{2}}(\mathbb{Z})}\left[\mathbf{M}_{s}(4 \pi|n| y) \mathfrak{e}_{\alpha}(n x)\right]\right|_{k}(M, \phi),
$$

where $\tau=x+i y \in \mathfrak{H}, s \in \mathbb{C}$ with $\operatorname{Re}(s)>1$ and $\widetilde{\Gamma}_{\infty}$ is the subgroup of $\operatorname{Mp}_{2}(\mathbb{Z})$ generated by $T$. Then we have

$$
\Phi_{\alpha, n}(Z, s)=\lim _{u \rightarrow \infty} \int_{\mathcal{F}_{u}}\left\langle F_{\alpha, n}(\tau, s), \Theta_{L}(\tau, Z)\right\rangle y \frac{d x d y}{y^{2}},
$$

where $\mathcal{F}_{u}=\{\tau=x+i y|| \tau|\geq 1| x \mid, \geq 1 / 2, y \leq u\}$ if $\operatorname{Re}(s)$ is sufficiently large ([Br2], 2.2 and 2.3). The additive symmetry for $\Phi_{\alpha, n}(Z, s)$ follows directly from that of $\Theta_{L}(\tau, Z)$. 


\section{Proof of Theorem 3.1}

\subsection{Reduction}

First note that, to prove Theorem [3.1, it suffices to show

$$
\begin{aligned}
& \Theta_{\alpha}\left(\tau,\left(p z, \sqrt{p} w, z^{\prime}\right)\right)+\sum_{a=0}^{p-1} \Theta_{\alpha}\left(\tau,\left(p^{-1}(z+a), \sqrt{p}^{-1} w, z^{\prime}\right)\right) \\
& \quad=\Theta_{\alpha}\left(\tau,\left(z, \sqrt{p} w, p z^{\prime}\right)\right)+\sum_{a=0}^{p-1} \Theta_{\alpha}\left(\tau,\left(z, \sqrt{p}^{-1} w, p^{-1}\left(z^{\prime}+a\right)\right)\right)
\end{aligned}
$$

for any $\alpha \in L^{*} / L$ and any prime $p$. Thrroughout this section, we fix an $\alpha \in L^{*} / L$ and a prime $p$. Take an $\alpha_{0} \in L_{0}^{*}$ such that

$$
\alpha \equiv\left(\begin{array}{c}
0 \\
0 \\
\alpha_{0} \\
0 \\
0
\end{array}\right)(\bmod L)
$$

and put

$$
\alpha_{1}=\left(0, \alpha_{0}, 0\right) \in L_{1}^{*} .
$$

Let $\mathcal{Y}=\left\{Y={ }^{t}\left(y_{1}, \ldots, y_{m+2}\right) \in V_{1} \mid Q_{1}[Y]>0, y_{1}>0\right\}$. Note that $\operatorname{Im}(Z) \in \mathcal{Y}$ for $Z \in \mathcal{D}$, and that $\mathcal{Y}$ is naturally identified with the Grassmannian of $L_{1}$. For $\tau \in \mathfrak{H}, Y \in \mathcal{Y}, r, t \in V_{1}$, we define the generalized Siegel theta function $\theta_{\alpha_{1}}$ for $L_{1}$ by

$$
\theta_{\alpha_{1}}(\tau, Y ; r, t)=\sum_{\lambda \in \alpha_{1}+L_{1}} \mathbf{e}\left(i \operatorname{Im}(\tau) \frac{Q_{1}(\lambda+t, Y)^{2}}{Q_{1}[Y]}+\frac{\bar{\tau}}{2} Q_{1}[\lambda+t]-Q_{1}\left(\lambda+\frac{t}{2}, r\right)\right) .
$$

The following formula due to Borcherds ([Bo2], Theorem 5.2; see also [Br], Theorem 2.4) plays a crucial role in the proof of Theorem 3.1.

Proposition 7.1. We have

$$
\Theta_{\alpha}(\tau, Z)=\sqrt{\frac{Q_{1}\left[Y_{Z}\right]}{2 \operatorname{Im}(\tau)}} \sum_{c, d \in \mathbb{Z}} \mathbf{e}\left(\frac{i|c \tau+d|^{2} Q_{1}\left[Y_{Z}\right]}{4 \operatorname{Im}(\tau)}\right) \theta_{\alpha_{1}}\left(\tau, Y_{Z} ; d X_{Z},-c X_{Z}\right),
$$

where $X_{Z}=\operatorname{Re}(Z)$ and $Y_{Z}=\operatorname{Im}(Z)$, and $\alpha_{1}$ as defined above.

From now on we fix $\tau \in \mathfrak{H}$ and $Z \in \mathcal{D}$. Let

$$
X=X_{Z}=\left(x, X_{0}, x^{\prime}\right), Y=Y_{Z}=\left(y, Y_{0}, y^{\prime}\right) \quad\left(x, x^{\prime} \in \mathbb{R}, y, y^{\prime} \in \mathbb{R}_{>0}, X_{0}, Y_{0} \in V_{0}=\mathbb{R}^{m}\right)
$$

and put

$$
\begin{aligned}
& X_{+}^{\uparrow}=\left(p x, \sqrt{p} X_{0}, x^{\prime}\right), \quad Y_{+}^{\uparrow}=\left(p y, \sqrt{p} Y_{0}, y^{\prime}\right), \\
& X_{-}^{\uparrow}(a)=\left(p^{-1}(x+a), \sqrt{p}^{-1} X_{0}, x^{\prime}\right), \quad Y_{-}^{\uparrow}=\left(p^{-1} y, \sqrt{p}^{-1} Y_{0}, y^{\prime}\right), \\
& X_{+}^{\downarrow}=\left(x, \sqrt{p} X_{0}, p x^{\prime}\right), \quad Y_{+}^{\downarrow}=\left(y, \sqrt{p} Y_{0}, p y^{\prime}\right), \\
& X_{-}^{\downarrow}(a)=\left(x, \sqrt{p}^{-1} X_{0}, p^{-1}\left(x^{\prime}+a\right)\right), \quad Y_{-}^{\downarrow}=\left(y, \sqrt{p}^{-1} Y_{0}, p^{-1} y^{\prime}\right)
\end{aligned}
$$


for $a \in \mathbb{Z}$. We set

$$
\begin{aligned}
I_{+}^{\uparrow}(c, d) & =\theta_{\alpha_{1}}\left(\tau, Y_{+}^{\uparrow} ; d X_{+}^{\uparrow},-c X_{+}^{\uparrow}\right), \\
I_{-}^{\uparrow}(c, d) & =\sum_{a=0}^{p-1} \theta_{\alpha_{1}}\left(\tau, Y_{-}^{\uparrow} ; d X_{-}^{\uparrow}(a),-c X_{-}^{\uparrow}(a)\right), \\
I_{+}^{\downarrow}(c, d) & =\theta_{\alpha_{1}}\left(\tau, Y_{+}^{\downarrow} ; d X_{+}^{\downarrow},-c X_{+}^{\downarrow}\right), \\
I_{-}^{\downarrow}(c, d) & =\sum_{a=0}^{p-1} \theta_{\alpha_{1}}\left(\tau, Y_{-}^{\downarrow} ; d X_{-}^{\downarrow}(a),-c X_{-}^{\downarrow}(a)\right)
\end{aligned}
$$

for $c, d \in \mathbb{Z}$. Observe that $Q_{1}\left[Y_{+}^{\uparrow}\right]=Q_{1}\left[Y_{+}^{\downarrow}\right]=p Q_{1}[Y]$ and $Q_{1}\left[Y_{-}^{\uparrow}\right]=Q_{1}\left[Y_{-}^{\downarrow}\right]=p^{-1} Q_{1}[Y]$. In view of Proposition 7.1, the proof of (7.1) is reduced to the following equality:

$$
\begin{aligned}
& \sum_{c, d \in \mathbb{Z}} \mathbf{e}\left(p \frac{i|c \tau+d|^{2} Q_{1}\left[Y_{Z}\right]}{4 \operatorname{Im}(\tau)}\right) I_{+}^{\uparrow}(c, d)+p^{-1} \sum_{c, d \in \mathbb{Z}} \mathbf{e}\left(p^{-1} \frac{i|c \tau+d|^{2} Q_{1}\left[Y_{Z}\right]}{4 \operatorname{Im}(\tau)}\right) I_{-}^{\uparrow}(c, d) \\
& =\sum_{c, d \in \mathbb{Z}} \mathbf{e}\left(p \frac{i|c \tau+d|^{2} Q_{1}\left[Y_{Z}\right]}{4 \operatorname{Im}(\tau)}\right) I_{+}^{\downarrow}(c, d)+p^{-1} \sum_{c, d \in \mathbb{Z}} \mathbf{e}\left(p^{-1} \frac{i|c \tau+d|^{2} Q_{1}\left[Y_{Z}\right]}{4 \operatorname{Im}(\tau)}\right) I_{-}^{\downarrow}(c, d)
\end{aligned}
$$

Theorem 7.2. (i) If $p \nmid m$ or $p \nmid n$, we have

$$
I_{-}^{\uparrow}(c, d)=I_{-}^{\downarrow}(c, d) .
$$

(ii) If $p \mid m$ and $p \mid n$, we have

$$
I_{-}^{\uparrow}(c, d)=p I_{+}^{\downarrow}\left(p^{-1} c, p^{-1} d\right)
$$

and

$$
I_{-}^{\downarrow}(c, d)=p I_{+}^{\uparrow}\left(p^{-1} c, p^{-1} d\right) .
$$

We now show that Theorem 7.2 implies the equality (7.3) and hence Theorem 3.1. By (7.4), the left-hand side of (7.3) is equal to

$$
\begin{aligned}
& \sum_{c, d \in \mathbb{Z}} \mathbf{e}\left(p \frac{i|c \tau+d|^{2} Q_{1}[Y]}{4 \operatorname{Im}(\tau)}\right) I_{+}^{\uparrow}(c, d)+\sum_{c, d \in \mathbb{Z}} \mathbf{e}\left(p \frac{i|c \tau+d|^{2} Q_{1}[Y]}{4 \operatorname{Im}(\tau)}\right) I_{+}^{\downarrow}(c, d) \\
& +p^{-1} \sum_{(c, d) \in \Lambda_{p}} \mathbf{e}\left(p^{-1} \frac{i|c \tau+d|^{2} Q_{1}[Y]}{4 \operatorname{Im}(\tau)}\right) I_{-}^{\uparrow}(c, d),
\end{aligned}
$$

where $\Lambda_{p}=\left\{(c, d) \in \mathbb{Z}^{2} \mid p \nmid m\right.$ or $\left.p \nmid n\right\}$. Similarly, by (7.6), the right-hand side of (17.3) is equal to

$$
\begin{aligned}
& \sum_{c, d \in \mathbb{Z}} \mathbf{e}\left(p \frac{i|c \tau+d|^{2} Q_{1}[Y]}{4 \operatorname{Im}(\tau)}\right) I_{+}^{\downarrow}(c, d)+\sum_{c, d \in \mathbb{Z}} \mathbf{e}\left(p \frac{i|c \tau+d|^{2} Q_{1}[Y]}{4 \operatorname{Im}(\tau)}\right) I_{+}^{\uparrow}(c, d) \\
& \quad+p^{-1} \sum_{(c, d) \in \Lambda_{p}} \mathbf{e}\left(p^{-1} \frac{i|c \tau+d|^{2} Q_{1}[Y]}{4 \operatorname{Im}(\tau)}\right) I_{-}^{\downarrow}(c, d) .
\end{aligned}
$$

The equality (7.3) now follows from (7.4). 


\subsection{Generalized Siegel theta functions}

To prove Theorem 7.2 , we need the following formulas for generalized Siegel theta functions.

Lemma 7.3. Let $X=\left(x, X_{0}, x^{\prime}\right) \in V_{1}$ and $Y=\left(y, Y_{0}, y^{\prime}\right) \in \mathcal{Y}$.

(i) We have

$$
\begin{aligned}
& \theta_{\alpha_{1}}(\tau, Y ; d X,-c X) \\
& =\mathbf{e}\left(\frac{c d}{2}\left(2 x x^{\prime}-S\left[X_{0}\right]\right)\right) \\
& \times \sum_{\substack{l, l^{\prime} \in \mathbb{Z} \\
\lambda_{0} \in \alpha_{0}+L_{0}}} \mathbf{e}\left(\frac{i \operatorname{Im}(\tau)}{Q_{1}[Y]}\left\{(l-c x) y^{\prime}+\left(l^{\prime}-c x^{\prime}\right) y-S\left(\lambda_{0}-c X_{0}, Y_{0}\right)\right\}^{2}\right. \\
& \left.\quad+\frac{\bar{\tau}}{2}\left(2(l-c x)\left(l^{\prime}-c x^{\prime}\right)-S\left[\lambda_{0}-c X_{0}\right]\right)-d\left(l x^{\prime}+l^{\prime} x-S\left(\lambda_{0}, X_{0}\right)\right)\right) .
\end{aligned}
$$

(ii) We have

$$
\begin{aligned}
& \theta_{\alpha_{1}}(\tau, Y ; d X,-c X) \\
& =\sqrt{\frac{Q_{1}[Y]}{2 y^{2} \operatorname{Im}(\tau)}} \mathbf{e}\left(\frac{c d}{2}\left(2 x x^{\prime}-S\left[X_{0}\right]\right)\right) \\
& \times \sum_{\substack{l \in \mathbb{Z} \\
\lambda_{0} \in \alpha_{0}+L_{0}}} \mathbf{e}\left(\frac{i \operatorname{Im}(\tau)}{Q_{1}[Y]}\left\{(l-c x) y^{\prime}-c x^{\prime} y-S\left(\lambda_{0}-c X_{0}, Y_{0}\right)\right\}^{2}\right. \\
& \left.+\frac{\bar{\tau}}{2}\left(-2 c x^{\prime}(l-c x)-S\left[\lambda_{0}-c X_{0}\right]\right)-d\left(l x^{\prime}-S\left(\lambda_{0}, X_{0}\right)\right)\right) \\
& \sum_{l^{\prime} \in \mathbb{Z}} \mathbf{e}\left(\frac { i Q _ { 1 } [ Y ] } { 4 y ^ { 2 } \operatorname { I m } ( \tau ) } \left\{l^{\prime}+\frac{2 i y \operatorname{Im}(\tau)}{Q_{1}[Y]}\left((l-c x) y^{\prime}-c x^{\prime} y-S\left(\lambda_{0}-c X_{0}, Y_{0}\right)\right)\right.\right. \\
& \left.+\bar{\tau}(l-c x)-d x\}^{2}\right)
\end{aligned}
$$

and

$$
\begin{aligned}
& \theta_{\alpha_{1}}(\tau, Y ; d X,-c X) \\
& =\sqrt{\frac{Q_{1}[Y]}{2\left(y^{\prime}\right)^{2} \operatorname{Im}(\tau)} \mathbf{e}\left(\frac{c d}{2}\left(2 x x^{\prime}-S\left[X_{0}\right]\right)\right)} \\
& \quad \times \sum_{\substack{l^{\prime} \in \mathbb{Z} \\
\lambda_{0} \in \alpha_{0}+L_{0}}} \mathbf{e}\left(\frac{i \operatorname{Im}(\tau)}{Q_{1}[Y]}\left\{-c x y^{\prime}+\left(l^{\prime}-c x^{\prime}\right) y-S\left(\lambda_{0}-c X_{0}, Y_{0}\right)\right\}^{2}\right. \\
& \left.\quad+\frac{\bar{\tau}}{2}\left(-2 c x\left(l^{\prime}-c x^{\prime}\right)-S\left[\lambda_{0}-c X_{0}\right]\right)-d\left(l^{\prime} x-S\left(\lambda_{0}, X_{0}\right)\right)\right) \\
& \sum_{l \in \mathbb{Z}} \mathbf{e}\left(\frac { i Q _ { 1 } [ Y ] } { 4 ( y ^ { \prime } ) ^ { 2 } \operatorname { I m } ( \tau ) } \left\{l+\frac{2 i y^{\prime} \operatorname{Im}(\tau)}{Q_{1}[Y]}\left(-c x y^{\prime}+\left(l^{\prime}-c x^{\prime}\right) y-S\left(\lambda_{0}-c X_{0}, Y_{0}\right)\right)\right.\right. \\
& \left.\left.+\bar{\tau}\left(l^{\prime}-c x^{\prime}\right)-d x^{\prime}\right\}^{2}\right) .
\end{aligned}
$$


Proof. The first assertion is immediate from (7.2). The equality (7.8) (respectively (7.9)) is obtained by applying the Poisson summation formula to the sum over $l^{\prime} \in \mathbb{Z}$ (respectively $l \in \mathbb{Z}$ ) in the right-hand side of (17.7).

\subsection{Proof of Theorem $\mathbf{7 . 2}$ (i)}

In this and the next subsections, we keep the notation of 7.1. In view of (7.7), we have

$$
\begin{aligned}
& I_{-}^{\uparrow}(c, d) \\
& =\sum_{\substack{l, l^{\prime} \in \mathbb{Z} \\
\lambda_{0} \in \alpha_{0}+L_{0}}} \sum_{a=0}^{p-1} \mathbf{e}\left(\frac{c d}{2}\left(2 p^{-1}(x+a) x^{\prime}-p^{-1} S\left[X_{0}\right]\right)\right. \\
& +p \frac{i \operatorname{Im}(\tau)}{Q_{1}[Y]}\left\{\left(l-p^{-1} c(x+a)\right) y^{\prime}+p^{-1}\left(l^{\prime}-c x^{\prime}\right) y-S\left(\lambda_{0}-c \sqrt{p}^{-1} X_{0}, \sqrt{p}-1 Y_{0}\right)\right\}^{2} \\
& +\frac{\bar{\tau}}{2}\left(2\left(l-p^{-1} c(x+a)\right)\left(l^{\prime}-c x^{\prime}\right)-S\left[\lambda_{0}-c \sqrt{p}^{-1} X_{0}\right]\right) \\
& \left.-d\left(l x^{\prime}+p^{-1} l^{\prime}(x+a)-S\left(\lambda_{0}, \sqrt{p}^{-1} X_{0}\right)\right)\right)
\end{aligned}
$$

and

$$
\begin{aligned}
& I_{-}^{\downarrow}(c, d) \\
& =\sum_{\substack{l, l^{\prime} \in \mathbb{Z} \\
\lambda_{0} \in \alpha_{0}+L_{0}}} \sum_{a=0}^{p-1} \mathbf{e}\left(\frac{c d}{2}\left(2 p^{-1} x\left(x^{\prime}+a\right)-p^{-1} S\left[X_{0}\right]\right)\right. \\
& +p \frac{i \operatorname{Im}(\tau)}{Q_{1}[Y]}\left\{p^{-1}(l-c x) y^{\prime}+\left(l^{\prime}-p^{-1} c\left(x^{\prime}+a\right)\right) y-S\left(\lambda_{0}-c \sqrt{p}^{-1} X_{0}, \sqrt{p}-1 Y_{0}\right)\right\}^{2} \\
& +\frac{\bar{\tau}}{2}\left(2(l-c x)\left(l^{\prime}-p^{-1} c\left(x^{\prime}+a\right)\right)-S\left[\lambda_{0}-c \sqrt{p}^{-1} X_{0}\right]\right) \\
& \left.-d\left(p^{-1} l\left(x^{\prime}+a\right)+l^{\prime} x-S\left(\lambda_{0}, \sqrt{p}^{-1} X_{0}\right)\right)\right) .
\end{aligned}
$$

First suppose that $p \mid c$ and $p \nmid d$. Changing $l$ into $l+p^{-1} c a$ in the sum (7.10), we obtain

$$
\begin{aligned}
I_{-}^{\uparrow}(c, d) & \\
= & \mathbf{e}\left(\frac{c d}{2 p}\left(2 x x^{\prime}-S\left[X_{0}\right]\right)\right) \\
& \sum_{\substack{l, l^{\prime} \in \mathbb{Z} \\
\lambda_{0} \in \alpha_{0}+L_{0}}} \mathbf{e}\left(p \frac{i \operatorname{Im}(\tau)}{Q_{1}[Y]}\left\{\left(l-p^{-1} c x\right) y^{\prime}+p^{-1}\left(l^{\prime}-c x^{\prime}\right) y-S\left(\lambda_{0}-c \sqrt{p}^{-1} X_{0}, \sqrt{p}^{-1} Y_{0}\right)\right\}^{2}\right. \\
& \left.\quad+\frac{\bar{\tau}}{2}\left(2\left(l-p^{-1} c x\right)\left(l^{\prime}-c x^{\prime}\right)-S\left[\lambda_{0}-c \sqrt{p}^{-1} X_{0}\right]\right)-d\left(l x^{\prime}+p^{-1} l^{\prime} x-S\left(\lambda_{0}, \sqrt{p}^{-1} X_{0}\right)\right)\right) \\
& \times \sum_{a=0}^{p-1} \mathbf{e}\left(-\frac{d l^{\prime}}{p} a\right) .
\end{aligned}
$$


Since the last sum is equal to $p \delta\left(p \mid l^{\prime}\right)$, we have

$$
\begin{aligned}
& I_{-}^{\uparrow}(c, d) \\
& =p \mathbf{e}\left(\frac{c d}{2 p}\left(2 x x^{\prime}-S\left[X_{0}\right]\right)\right) \\
& \quad \sum_{\substack{l, l^{\prime} \in \mathbb{Z} \\
\lambda_{0} \in \alpha_{0}+L_{0}}} \mathbf{e}\left(p \frac{i \operatorname{Im}(\tau)}{Q_{1}[Y]}\left\{\left(l-p^{-1} c x\right) y^{\prime}+\left(l^{\prime}-p^{-1} c x^{\prime}\right) y-S\left(\lambda_{0}-c \sqrt{p}^{-1} X_{0}, \sqrt{p}^{-1} Y_{0}\right)\right\}^{2}\right. \\
& \left.\quad+\frac{\bar{\tau}}{2}\left(2 p\left(l-p^{-1} c x\right)\left(l^{\prime}-p^{-1} c x^{\prime}\right)-S\left[\lambda_{0}-c \sqrt{p}^{-1} X_{0}\right]\right)-d\left(l x^{\prime}+l^{\prime} x-S\left(\lambda_{0}, \sqrt{p}^{-1} X_{0}\right)\right)\right) .
\end{aligned}
$$

A similar calculation shows that $I_{-}^{\downarrow}(c, d)$ is equal to the right-hand side of the above equality. Thus the equality (7.4) has been proved in the case $p \mid c$ and $p \nmid d$.

Next suppose that $p \nmid c$. Then $l_{1}=p l-c a$ runs over $\mathbb{Z}$ as $l$ runs over $\mathbb{Z}$ and $a$ over $\{0,1, \ldots, p-1\}$. Take an integer $c_{0}$ such that $c c_{0} \equiv 1(\bmod p)$. Then $a \equiv-c_{0} l_{1}(\bmod p)$. In view of (7.10), we have

$$
\begin{aligned}
& I_{-}^{\uparrow}(c, d) \\
& =\mathbf{e}\left(\frac{c d}{2 p}\left(2 x x^{\prime}-S\left[X_{0}\right]\right)\right) \\
& \quad \sum_{\substack{l_{1}, l^{\prime} \in \mathbb{Z} \\
\lambda_{0} \in \alpha_{0}+L_{0}}} \mathbf{e}\left(p \frac{i \operatorname{Im}(\tau)}{Q_{1}[Y]}\left\{p^{-1}\left(l_{1}-c x\right) y^{\prime}+p^{-1}\left(l^{\prime}-c x^{\prime}\right) y-S\left(\lambda_{0}-c \sqrt{p}^{-1} X_{0}, \sqrt{p}^{-1} Y_{0}\right)\right\}^{2}\right. \\
& \quad+\frac{\bar{\tau}}{2}\left(2 p^{-1}\left(l_{1}-c x\right)\left(l^{\prime}-c x^{\prime}\right)-S\left[\lambda_{0}-c \sqrt{p}^{-1} X_{0}\right]\right)-d\left(p^{-1} l_{1} x^{\prime}+p^{-1} l^{\prime} x-S\left(\lambda_{0}, \sqrt{p}^{-1} X_{0}\right)\right) \\
& \left.\quad+p^{-1} c_{0} d l_{1} l^{\prime}\right) .
\end{aligned}
$$

On the other hand, since $l_{1}^{\prime}=p l^{\prime}-c a$ runs over $\mathbb{Z}$ as $l$ runs over $\mathbb{Z}$ and $a$ over $\{0,1, \ldots, p-1\}$, and since $a \equiv-c_{0} l_{1}^{\prime}(\bmod p)$, we obtain

$$
\begin{aligned}
& I_{-}^{\downarrow}(c, d) \\
& =\mathbf{e}\left(\frac{c d}{2 p}\left(2 x x^{\prime}-S\left[X_{0}\right]\right)\right) \\
& \quad \sum_{\substack{l, l_{1}^{\prime} \in \mathbb{Z} \\
\lambda_{0} \in \alpha_{0}+L_{0}}} \mathbf{e}\left(p \frac{i \operatorname{Im}(\tau)}{Q_{1}[Y]}\left\{p^{-1}(l-c x) y^{\prime}+p^{-1}\left(l_{1}^{\prime}-c x^{\prime}\right) y-S\left(\lambda_{0}-c \sqrt{p}^{-1} X_{0}, \sqrt{p}^{-1} Y_{0}\right)\right\}^{2}\right. \\
& \quad+\frac{\bar{\tau}}{2}\left(2 p^{-1}(l-c x)\left(l_{1}^{\prime}-c x^{\prime}\right)-S\left[\lambda_{0}-c \sqrt{p}^{-1} X_{0}\right]\right)-d\left(p^{-1} l x^{\prime}+p^{-1} l_{1}^{\prime} x-S\left(\lambda_{0}, \sqrt{p}^{-1} X_{0}\right)\right) \\
& \left.\quad+p^{-1} c_{0} d l l_{1}^{\prime}\right) .
\end{aligned}
$$

Comparing these two expressions, we obtain the equality (7.4) in the case $p \nmid c$. 


\subsection{Proof of Theorem 7.2 (ii)}

Suppose that $p \mid c$ and $p \mid d$. By (7.8), we have

$$
\begin{aligned}
& I_{-}^{\uparrow}(c, d) \\
& =\sqrt{p} \sqrt{\frac{Q_{1}[Y]}{2 y^{2} \operatorname{Im}(\tau)} \sum_{a=0}^{p-1} \mathbf{e}\left(\frac{c d}{2}\left(2 p^{-1}(x+a) x^{\prime}-p^{-1} S\left[X_{0}\right]\right)\right)} \\
& \sum_{\substack{l \in \mathbb{Z} \\
\lambda_{0} \in \alpha_{0}+L_{0}}} \mathbf{e}\left(p \frac{i \operatorname{Im}(\tau)}{Q_{1}[Y]}\left\{\left(l-p^{-1} c(x+a)\right) y^{\prime}-p^{-1} c x^{\prime} y-S\left(\lambda_{0}-c \sqrt{p}^{-1} X_{0}, \sqrt{p}^{-1} Y_{0}\right)\right\}^{2}\right. \\
& \left.\quad+\frac{\bar{\tau}}{2}\left(-2 c x^{\prime}\left(l-p^{-1} c(x+a)\right)-S\left[\lambda_{0}-c \sqrt{p}^{-1} X_{0}\right]\right)-d\left(l x^{\prime}-S\left(\lambda_{0}, \sqrt{p}^{-1} X_{0}\right)\right)\right) \\
& \sum_{l^{\prime} \in \mathbb{Z}} \mathbf{e}\left(p \frac { i Q _ { 1 } [ Y ] } { 4 y ^ { 2 } \operatorname { I m } ( \tau ) } \left\{l^{\prime}+\frac{2 i y \operatorname{Im}(\tau)}{Q_{1}[Y]}\left(\left(l-p^{-1} c(x+a)\right) y^{\prime}-p^{-1} c x^{\prime} y-S\left(\lambda_{0}-c \sqrt{p}^{-1} X_{0}, \sqrt{p}^{-1} Y_{0}\right)\right)\right.\right. \\
& \left.\left.+\bar{\tau}\left(l-p^{-1} c(x+a)\right)-p^{-1} d(x+a)\right\}^{2}\right) .
\end{aligned}
$$

Changing $l$ into $l+p^{-1} c a$ and $l^{\prime}$ into $l^{\prime}+p^{-1} d a$ respectively, we see that $I_{-}^{\uparrow}(c, d)$ is equal to

$$
\begin{aligned}
& p \sqrt{p} \sqrt{\frac{Q_{1}[Y]}{2 y^{2} \operatorname{Im}(\tau)}} \mathbf{e}\left(\frac{c d}{2 p}\left(2 x x^{\prime}-S\left[X_{0}\right]\right)\right) \\
& \times \sum_{\substack{l \in \mathbb{Z} \\
\lambda_{0} \in \alpha_{0}+L_{0}}} \mathbf{e}\left(p \frac{i \operatorname{Im}(\tau)}{Q_{1}[Y]}\left\{\left(l-p^{-1} c x\right) y^{\prime}-p^{-1} c x^{\prime} y-S\left(\lambda_{0}-c \sqrt{p}^{-1} X_{0}, \sqrt{p}^{-1} Y_{0}\right)\right\}^{2}\right. \\
& \left.+\frac{\bar{\tau}}{2}\left(-2 c x^{\prime}\left(l-p^{-1} c x\right)-S\left[\lambda_{0}-c \sqrt{p}^{-1} X_{0}\right]\right)-d\left(l x^{\prime}-S\left(\lambda_{0}, \sqrt{p}^{-1} X_{0}\right)\right)\right) \\
& \sum_{l^{\prime} \in \mathbb{Z}} \mathbf{e}\left(p \frac { i Q _ { 1 } [ Y ] } { 4 y ^ { 2 } \operatorname { I m } ( \tau ) } \left\{l^{\prime}+p^{-1} \frac{2 i y \operatorname{Im}(\tau)}{Q_{1}[Y]}\left(p\left(l-p^{-1} c x\right) y^{\prime}-c x^{\prime} y-S\left(\lambda_{0}-c \sqrt{p}^{-1} X_{0}, \sqrt{p} Y_{0}\right)\right)\right.\right. \\
& \left.\left.+\bar{\tau}\left(l-p^{-1} c x\right)-p^{-1} d x\right\}^{2}\right) .
\end{aligned}
$$


Using again (7.8), we see that $p I_{+}^{\downarrow}\left(p^{-1} c, p^{-1} d\right)$ is equal to

$$
\begin{gathered}
p \sqrt{p} \sqrt{\frac{Q_{1}[Y]}{2 y^{2} \operatorname{Im}(\tau)} \mathbf{e}\left(\frac{c d}{2 p}\left(2 x x^{\prime}-S\left[X_{0}\right]\right)\right)} \\
\times \sum_{\substack{l \in \mathbb{Z} \\
\lambda_{0} \in \alpha_{0}+L_{0}}} \mathbf{e}\left(p^{-1} \frac{i \operatorname{Im}(\tau)}{Q_{1}[Y]}\left\{\left(l-\left(p^{-1} c\right) x\right) p y^{\prime}-\left(p^{-1} c\right) p x^{\prime} y-S\left(\lambda_{0}-\left(p^{-1} c\right) \sqrt{p} X_{0}, \sqrt{p} Y_{0}\right)\right\}^{2}\right. \\
\left.\quad+\frac{\bar{\tau}}{2}\left(-2\left(p^{-1} c\right) p x^{\prime}\left(l-\left(p^{-1} c\right) x\right)-S\left[\lambda_{0}-\left(p^{-1} c\right) \sqrt{p} X_{0}\right]\right)-p^{-1} d\left(l p x^{\prime}-S\left(\lambda_{0}, \sqrt{p} X_{0}\right)\right)\right) \\
\sum_{l^{\prime} \in \mathbb{Z}} \mathbf{e}\left(p \frac { i Q _ { 1 } [ Y ] } { 4 y ^ { 2 } \operatorname { I m } ( \tau ) } \left\{l^{\prime}+p^{-1} \frac{2 i y \operatorname{Im}(\tau)}{Q_{1}[Y]}\left(\left(l-\left(p^{-1} c\right) x\right) p y^{\prime}-\left(p^{-1} c\right) p x^{\prime} y\right.\right.\right. \\
\left.\left.\left.-S\left(\lambda_{0}-\left(p^{-1} c\right) \sqrt{p} X_{0}, \sqrt{p} Y_{0}\right)\right)+\bar{\tau}\left(l-\left(p^{-1} c\right) x\right)-\left(p^{-1} d\right) x\right\}^{2}\right) .
\end{gathered}
$$

Comparing (7.12) and (7.13), we obtain the equality (7.5). The equality (7.6) is proved in a similar manner. Then the proof of Theorem 7.2 has been completed.

\section{Examples}

\subsection{Siegel modular forms of degree two}

In this section, we consider the case where $m=1$ and $S=(2)$. In this case, $\mathcal{D}$ is isomorphic to the Siegel upper half space $\mathfrak{H}_{2}$ of degree 2, and the space of holomorphic automorphic forms on $\Gamma^{*}(L)$ of weight $k$ is naturally identified with the space $M_{k}\left(\Gamma_{2}\right)$ of holomorphic Siegel modular forms on $\Gamma_{2}=\operatorname{Sp}_{2}(\mathbb{Z})$ of weight $k$. We denote by $S_{k}\left(\Gamma_{2}\right)$ the space of cusp forms in $M_{k}\left(\Gamma_{2}\right)$.

It is known that $S_{10}\left(\Gamma_{2}\right)$ and $S_{12}\left(\Gamma_{2}\right)$ are one dimensional. Let $\chi_{10}$ and $\chi_{12}$ be nonzero elements of $S_{10}\left(\Gamma_{2}\right)$ and $S_{12}\left(\Gamma_{2}\right)$ respectively. Then $\chi_{10}$ is a Borcherds lift ([GN]) and hence satisfies the multiplicative symmetry. We will show that, on the other hand, $\chi_{12}$ does not satisfy the multiplicative symmetry and hence is not a Borcherds lift.

\subsection{The Saito-Kurokawa lifting}

To calculate the Fourier coefficients of $\chi_{10}$ and $\chi_{12}$, it is convenient to express them as SaitoKurokawa lifts (see [EZ]). Let $k, m \in \mathbb{N}$. For a holomorphic function $\phi$ on $\mathfrak{H} \times \mathbb{C}$, we put

$$
\begin{aligned}
\left(\left.\phi\right|_{k . m} \gamma\right)(\tau, z) & =(c \tau+d)^{-k} \mathbf{e}\left(m \frac{-c z^{2}}{c \tau+d}\right) \phi\left(\frac{a \tau+b}{c \tau+d}, \frac{z}{c \tau+d}\right) \quad\left(\gamma=\left(\begin{array}{cc}
a & b \\
c & d
\end{array}\right) \in \mathrm{SL}_{2}(\mathbb{R})\right), \\
\left(\left.\phi\right|_{m}[\lambda, \mu]\right)(\tau, z) & =\mathbf{e}\left(-m\left(\lambda^{2} \tau+2 \lambda z\right)\right) \phi(\tau, z+\lambda \tau+\mu) \quad(\lambda, \mu \in \mathbb{R}) .
\end{aligned}
$$

Let $J_{k, m}$ be the space of holomorphic functions $\phi$ on $\mathfrak{H} \times \mathbb{C}$ satifying

$$
\begin{aligned}
& \left.\phi\right|_{k, m} \gamma=\phi \quad\left(\gamma \in \mathrm{SL}_{2}(\mathbb{Z})\right), \\
& \left.\phi\right|_{m}[\lambda, \mu]=\phi \quad(\lambda, \mu \in \mathbb{Z})
\end{aligned}
$$


and $c_{\phi}(n, r)=0$ unless $4 n m \geq r^{2}$, where $\phi(\tau, z)=\sum_{n, r \in \mathbb{Z}} c_{\phi}(n, r) \mathbf{e}(n \tau+r z)$ is the Fourier expansion of $\phi$. We call $J_{k, m}$ the space of holomorphic Jacobi forms of weight $k$ and index $m$. Let $J_{k, 1}^{\text {cusp }}=\left\{\phi \in J_{k, m} \mid c_{\phi}(n, r)=0\right.$ unless $\left.4 n m>r^{2}\right\}$ be the space of Jacobi cusp forms of weight $k$ and index $m$. For $\phi \in J_{k, 1}^{\text {cusp }}$ and $m \in \mathbb{Z}_{>0}$, we put

$$
\left(\phi \mid V_{m}\right)(\tau, z)=m^{k-1} \sum_{\xi}(c \tau+d)^{-k} \mathbf{e}\left(m \frac{-c z^{2}}{c \tau+d}\right) \phi\left(\frac{a \tau+b}{c \tau+d}, \frac{z}{c \tau+d}\right),
$$

where $\xi=\left(\begin{array}{ll}a & b \\ c & d\end{array}\right)$ runs over $\mathrm{SL}_{2}(\mathbb{Z}) \backslash \mathrm{M}_{2}(\mathbb{Z})$ with $\operatorname{det} \xi=m$. Then $\phi \mid V_{m} \in J_{k, m}^{\text {cusp }}$. In what follows, we write $\left(\tau, z, \tau^{\prime}\right)$ for $\left(\begin{array}{cc}\tau & z \\ z & \tau^{\prime}\end{array}\right) \in \mathfrak{H}_{2}$. Define

$$
\mathcal{V}(\phi)\left(\tau, z, \tau^{\prime}\right)=\sum_{m=1}^{\infty}\left(\phi \mid V_{m}\right)(\tau, z) \mathbf{e}\left(m \tau^{\prime}\right) \quad\left(\left(\tau, z, \tau^{\prime}\right) \in \mathfrak{H}_{2}\right)
$$

The Saito-Kurokawa lift $\mathcal{V}(\phi)$ belongs to $S_{k}\left(\Gamma_{2}\right)$ and its Fourier expansion is given by

$$
\mathcal{V}(\phi)\left(\tau, z, \tau^{\prime}\right)=\sum_{n, r, m \in \mathbb{Z}, 4 n m>r^{2}, n>0} A(n, r, m) \mathbf{e}\left(n \tau+r z+m \tau^{\prime}\right)
$$

where

$$
A(n, r, m)=\sum_{0<d \mid(n, r, m)} d^{k-1} c_{\phi}\left(n m / d^{2}, r / d\right)
$$

(see [EZ $\S 3$ ).

\subsection{The Siegel modular forms $\chi_{10}$ and $\chi_{12}$}

For $k \in \mathbb{Z}$ with $k \geq 4$, put

$$
\begin{aligned}
E_{k}(\tau) & =\frac{1}{2} \sum_{c, d \in \mathbb{Z},(c, d)=1}(c \tau+d)^{-k} \quad(\tau \in \mathfrak{H}), \\
E_{k, 1}(\tau, z) & =\frac{1}{2} \sum_{c, d \in \mathbb{Z},(c, d)=1} \sum_{\lambda \in \mathbb{Z}}(c \tau+d)^{-k} \mathbf{e}\left(\lambda^{2} \frac{a \tau+b}{c \tau+d}+2 \lambda \frac{z}{c \tau+d}-\frac{c z^{2}}{c \tau+d}\right) \quad((\tau, z) \in \mathfrak{H} \times \mathbb{C}) .
\end{aligned}
$$

Then $E_{k} \in M_{k}\left(\mathrm{SL}_{2}(\mathbb{Z})\right)$ and $E_{k, 1} \in J_{k, 1}$. Set

$$
\begin{aligned}
\phi_{10,1}= & \frac{1}{144}\left(E_{6}(\tau) E_{4,1}(\tau, z)-E_{4}(\tau) E_{6,1}(\tau, z)\right) \\
= & \left(\zeta-2+\zeta^{-1}\right) q+\left(-2 \zeta^{2}-16 \zeta+36-16 \zeta^{-1}-2 \zeta^{-2}\right) q^{2} \\
& +\left(\zeta^{3}+36 \zeta^{2}+99 \zeta-272+99 \zeta^{-1}+36 \zeta^{-2}+\zeta^{-3}\right) q^{3}+\cdots, \\
\phi_{12,1}= & \frac{1}{144}\left(E_{4}(\tau)^{2} E_{4,1}(\tau, z)-E_{6}(\tau) E_{6,1}(\tau, z)\right) \\
= & \left(\zeta+10+\zeta^{-1}\right) q+\left(10 \zeta^{2}-88 \zeta-132-88 \zeta^{-1}+10 \zeta^{-2}\right) q^{2} \\
& +\left(\zeta^{3}-132 \zeta^{2}+1275 \zeta+736+1275 \zeta^{-1}-132 \zeta^{-2}+\zeta^{-3}\right) q^{3}+\cdots,
\end{aligned}
$$


where $q=\mathbf{e}(\tau), \zeta=\mathbf{e}(z)$. Then $\phi_{10,1} \in J_{10,1}^{\text {cusp }}$ and $\phi_{12,1} \in J_{12,1}^{\text {cusp }}$. Let $\chi_{10}=\mathcal{V}\left(\phi_{10,1}\right)$ and $\chi_{12}=\mathcal{V}\left(\phi_{12,1}\right)$ be the Saito-Kurokawa lifts of $\phi_{10,1}$ and $\phi_{12,1}$ respectively. It is known that $S_{10}\left(\Gamma_{2}\right)=\mathbb{C} \chi_{10}$ and $S_{12}\left(\Gamma_{2}\right)=\mathbb{C} \chi_{12}$, and that $\chi_{10}$ is a Borcherds lift.

\subsection{Relations satisfied by Fourier coefficients of Borcherds lifts}

Let

$$
F\left(\tau, z, \tau^{\prime}\right)=\sum_{n, r, m} A_{F}(n, r, m) \mathbf{e}\left(n \tau+r z+m \tau^{\prime}\right) \in S_{k}\left(\Gamma_{2}\right) .
$$

We make a convention that $A_{F}(n, r, m)=0$ unless $4 n m>r^{2}$ and $n>0$. Put

$$
\begin{aligned}
F^{\uparrow}\left(\tau, z, \tau^{\prime}\right) & =F \mid \mathcal{T}_{2}^{\uparrow}\left(\tau, \sqrt{2} z, \tau^{\prime}\right) \\
& =F\left(2 \tau, 2 z, \tau^{\prime}\right) F\left(\tau / 2, z, \tau^{\prime}\right) F\left((\tau+1) / 2, z, \tau^{\prime}\right)
\end{aligned}
$$

and

$$
\begin{aligned}
F^{\downarrow}\left(\tau, z, \tau^{\prime}\right) & =F \mid \mathcal{T}_{2}^{\downarrow}\left(\tau, \sqrt{2} z, \tau^{\prime}\right) \\
& \left.=F\left(\tau, 2 z, 2 \tau^{\prime}\right) F\left(\tau, z, \tau^{\prime} / 2\right) F\left(\tau, z,\left(\tau^{\prime}+1\right) / 2\right)\right) .
\end{aligned}
$$

Let

$$
\begin{aligned}
& F^{\uparrow}\left(\tau, z, \tau^{\prime}\right)=\sum_{n, r, m} A_{F}^{\uparrow}(n, r, m) \mathbf{e}\left(n \tau+r z+m \tau^{\prime}\right), \\
& F^{\downarrow}\left(\tau, z, \tau^{\prime}\right)=\sum_{n, r, m} A_{F}^{\downarrow}(n, r, m) \mathbf{e}\left(n \tau+r z+m \tau^{\prime}\right)
\end{aligned}
$$

be the Fourier expansions of $F^{\uparrow}$ and $F^{\downarrow}$ respectively.

Lemma 8.1. Suppose that $F$ is a Borcherds lift. Then there exists a complex number $\epsilon$ of absolute value 1 such that $A_{F}^{\uparrow}(n, r, m)=\epsilon A_{F}^{\downarrow}(n, r, m)$ for every $n, r, m$.

Proof. This follows from Theorem 5.3 .

A straightforward calculation shows the following:

Lemma 8.2. Let $F \in S_{k}\left(\Gamma_{2}\right)$. For $r \in \mathbb{Z}$, we have

$$
\begin{aligned}
& A_{F}^{\uparrow}(4, r, 3)=\sum_{r_{1}, r_{2}, r_{3} \in \mathbb{Z}, 2 r_{1}+r_{2}+r_{3}=r} A_{F}\left(1, r_{1}, 1\right)\left\{A_{F}\left(2, r_{2}, 1\right) A_{F}\left(2, r_{3}, 1\right)\right. \\
& \left.-A_{F}\left(3, r_{2}, 1\right) A_{F}\left(1, r_{3}, 1\right)-A_{F}\left(1, r_{2}, 1\right) A_{F}\left(3, r_{3}, 1\right)\right\}
\end{aligned}
$$

and

$$
\begin{aligned}
A_{F}^{\downarrow}(4, r, 3)= & \sum_{\substack{\left.r_{1}, r_{2}, r_{3} \in \mathbb{Z}, 2 r_{1}+r_{2}+r_{3}=r \\
-A_{F}\left(1, r_{1}, 1\right) A_{F}\left(2, r_{2}, 1\right) A_{F}\left(1, r_{3}, 1\right)-A_{F}\left(1, r_{1}, 1\right) A_{F}\left(1, r_{2}, 1\right) A_{F}\left(2, r_{3}, 1\right)\right\} .}}\left\{-A_{F}\left(2, r_{1}, 1\right) A_{F}\left(1, r_{2}, 1\right) A_{F}\left(1, r_{3}, 1\right)\right. \\
&
\end{aligned}
$$


The values of $A_{F}^{\uparrow}(4, r, 3)$ and $A_{F}^{\downarrow}(4, r, 3)$ for several $r$ with $F=\chi_{10}$ and $F=\chi_{12}$ are given as follows.

\begin{tabular}{|c|c|c|c|c|}
\hline$r$ & 0 & 1 & 2 & 3 \\
\hline \hline$A_{\chi_{10}}^{\uparrow}(4, r, 3)$ & -552 & 216 & 222 & -212 \\
\hline$A_{\chi_{10}}^{\downarrow}(4, r, 3)$ & -552 & 216 & 222 & -212 \\
\hline \hline$A_{\chi_{12}}^{\uparrow}(4, r, 3)$ & 143304 & -59112 & 65310 & -20396 \\
\hline$A_{\chi_{12}}^{\downarrow}(4, r, 3)$ & 43512 & 26424 & 11850 & 3364 \\
\hline
\end{tabular}

This table together with Lemma 8.1 show the following:

Theorem 8.3. The Siegel cusp form $\chi_{12}$ is not a Borcherds lift.

\section{References}

[Bo1] R. E. Borcherds, Automorphic forms on $O_{s+2,2}(\mathbb{R})$ and infinite products, Invent. Math. 120 (1995), 161-213.

[Bo2] R. E. Borcherds, Automorphic forms with singularities on Grassmannians, Invent. Math. 132 (1998), 491-562.

[Br1] J. H. Bruinier, Borcherds products and Chern classes of Hirzeburch-Zagier divisors, Invent. Math. 138 (1999), 51-83.

[Br2] J. H. Bruinier, Borcherds Products on $O(2, l)$ and Chern Classes of Heegner Divisors, Lecture Notes in Math. 1780 (2002), Springer Verlag.

[BK] J. H. Bruinier and U. Kühn, Integrals of automorphic Green's functions associated to Heegner divisors, Int. Math. Res. Notices (2003) No. 31, 1687-1729.

[EZ] M. Eichler and D. Zagier, The theory of Jacobi forms (1985), Springer Verlag.

[GN] V. A. Gritsenko and V. V. Nikulin, Siegel automorphic form correction of some Lorentzian Kac-Moody Lie algebra, Amer. J. Math. 119 (1997), 181-224.

[GZ] B. Gross and D. Zagier, Heegner points and derivatives of L-series, Invent. Math. 84 (1986), no. 2, 225-320.

[HaMo] J. A. Harvey and G. Moore, Algebras, BPS states, and strings, Nuclear Physics B 463 (1996), 315-368.

[H] B. Heim, On the Spezialschar of Maass, arXiv:0801.1804. 
[HeMu] B. Heim and A. Murase, A characterization of the Maass space on $O(2, m+2)$ by symmetries, arXiv:1003.0573.

[HZ] F. Hirzeburch and D. Zagier, Intersection numbers of curves on Hilbert modular surfaces and modular forms of Nebentypus, Invent. Math. 36 (1976), 57-113.

[OT] T. Oda and M. Tsuzuki, Automorphic Green functions associated with the secondary spherical functions, Publ. Res. Inst. Math. Sci. 39 (2003), no. 3, 451-533.

[Si] C. L. Siegel, Indefinite quadratische Formen und Funktionentheorie I., Math. Ann. 124 (1951), 17-54.

[vdG] G. van der Geer, Hirbert modular surfaces, Ergebnisse der mathematik und ihre Grenzgebiete (3), 16, Springer-Verlag, Berlin, 1988.

Bernhard Heim

German University of Technology in Oman, Way No. 36, Building No. 331, North Ghubrah, Muscat, Sultanate of Oman

e-mail: bernhard.heim@gutech.edu.om

Atsushi Murase

Department of Mathematics, Faculty of Science, Kyoto Sangyo University, Motoyama, Kamigamo, Kita-ku, Kyoto 603-8555, Japan

e-mail: murase@cc.kyoto-su.ac.jp 